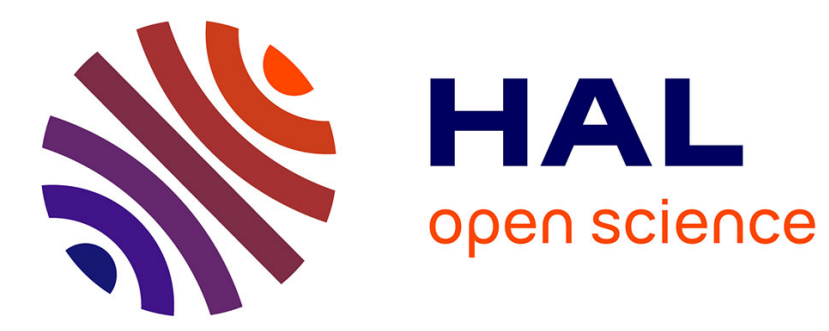

\title{
Dépouillement d'expériences de physique nucléaire à basse énergie
}

D. Daronian, A. Veyrié-Faucon

\section{To cite this version:}

D. Daronian, A. Veyrié-Faucon. Dépouillement d'expériences de physique nucléaire à basse énergie. Revue de Physique Appliquée, 1969, 4 (2), pp.137-138. 10.1051/rphysap:0196900402013700 . jpa00243182

\section{HAL Id: jpa-00243182 https://hal.science/jpa-00243182}

Submitted on 1 Jan 1969

HAL is a multi-disciplinary open access archive for the deposit and dissemination of scientific research documents, whether they are published or not. The documents may come from teaching and research institutions in France or abroad, or from public or private research centers.
L'archive ouverte pluridisciplinaire HAL, est destinée au dépôt et à la diffusion de documents scientifiques de niveau recherche, publiés ou non, émanant des établissements d'enseignement et de recherche français ou étrangers, des laboratoires publics ou privés. 


\title{
DÉPOUILLEMENT D'EXPÉRIENGES DE PHYSIQUE NUGLÉAIRE A BASSE ÉNERGIE
}

\author{
D. DARONIAN et A. VEYRIÉ-FAUCON, \\ Service de Physique Nucléaire à Basse Énergie, C.E.N., Saclay.
}

\begin{abstract}
Résumé. - Description sommaire d'une unité de traitement d'expériences de physique nucléaire, construite sur la base d'un calculateur CAE 510. Deux types de dépouillement sont explicités, mettant en évidence l'importance de périphériques non standarl tels que l'unité de visualisation et le traceur digital.
\end{abstract}

Abstract. - A nuclear handling system built around a CAE 510 computer is described. Two kinds of experiments, processed by this system, are shown, so that one can demonstrate the importance of the display unit and of the plotter in connexion with the computer.

Le calculateur CAE 510, installé auprès du Van de Graaff Tandem de Saclay, travaille actuellement à temps complet. L'automatisation partielle du système a permis de s'affranchir de la présence permanente de l'opérateur et parfois même de celle du physicien. Autant que possible, les heures de nuit sont consacrées à la partie du dépouillement qui a pu être automatisée, tandis que les journées le sont à la mise au point de nouveaux programmes, au dépouillement d'expériences demandant la présence du physicien et à la préparation du travail de nuit.

Le système CAE 510 que nous possédons est assez bien adapté au traitement d'expériences mono- ou multi-paramétriques autant du point de vue des entrées-sorties que de celui de l'appel de programmes en mémoire centrale. Une console d'exploitation [1] munie de voyants de contrôle et de boutons-poussoirs permet d'appeler en mémoire centrale et de lancer l'exécution de programmes de base par l'intermédiaire d'un programme moniteur. Tous ces programmes de base ainsi que le programme-moniteur sont stockés sur bande magnétique.

Une unité interface (CAE 592) permet le transfert de données intégrées à partir d'un bloc mémoire autonome, ou celui de données individuelles à partir d'un rythmeur (" derandomizer ») vers la mémoire du calculateur.

Trois dérouleurs de bande magnétique donnent une grande souplesse à l'installation : l'un de ces dérouleurs supporte la bande "moniteur ", les deux autres sont consacrés aux bandes-données et bandes-résultats. Il sera ainsi possible de travailler sans avoir à charger ou décharger des bandes en cours d'exécution de programme.

En dehors des périphériques standard tels que machine à écrire, lecteur-perforateur de ruban, lecteurperforateur de cartes, imprimante rapide, il faut signaler la présence d'un traceur Calcomp (300 incr/s, pas de $0,25 \mathrm{~mm}$ ) et celle d'une unité de visualisation RG 98 Intertechnique dotée d'un crayon sensible. Ce sont d'ailleurs ces deux périphériques spéciaux qui nous ont permis de mettre au point des méthodes de dépouillement originales.

Deux types principaux de dépouillement sont traités à l'aide du calculateur, l'un faisant intervenir la notion de rapidité et la facilité d'emploi des moyens de communication homme-machine (visualisation), l'autre concernant plutôt des dépouillements où le nombre et la complexité des opérations à effectuer rendent impossible toute exécution manuelle.

1) Dans les expériences du type « analyse de spectres mono-paramétriques ", les informations obtenues à partir d'un ou plusieurs détecteurs sont incrémentées dès le départ dans un bloc mémoire autonome (BM 96 Intertechnique). Dans certains cas, les informations individuelles, généralement stockées au préalable sur bande magnétique autonome d'un dérouleur à 16 pistes [2], sont incrémentées directement en mémoirecalculateur (16 k disponibles). Lorsque l'incrémentation se fait dans un bloc mémoire extérieur, le contenu de ce bloc est transféré, en fin d'expérience, dans la mémoire du calculateur. D'une part, une visualisation de la zone de mémoire correspondante sert à vérifier le bon déroulement de l'opération, et le tracé en histogramme du spectre obtenu permet de conserver une vue d'ensemble des résultats expérimentaux; d'autre part, un transfert de ces résultats sur bande magnétique compatible IBM établit la liaison avec le centre de calcul pour un dépouillement théorique ultérieur.

Un programme se servant de l'unité de visualisation comme organe de communication, à l'aide du crayon sensible, effectue un certain nombre d'opérations sur le spectre expérimental et extrait immédiatement une série de résultats préliminaires : lissage, soustraction du bruit de fond, calcul du barycentre des pics, construction d'une courbe d'étalonnage et extraction 
des énergies d'excitation. Après toute opération partielle, il est possible de comparer, à l'aide de la visualisation, le spectre initial et le spectre final; d'autre part, toutes les données et tous les résultats relatifs à ces différentes opérations sont imprimés sur machine à écrire afin de conserver un compte rendu des opérations effectuées.

Ce dépouillement immédiat devra être rapide, même si pour cela il faut sacrifier quelque peu la précision des résultats, car la connaissance immédiate de valeurs, même approchées, en cours de manipulation, s'avère suffisante pour prendre des décisions pouvant modifier le déroulement de l'expérience. La facilité d'emploi du crayon sensible comme moyen de dialogue entre le physicien et le calculateur rend ce dispositif indispensable; il faut cependant regretter que, sur l'unité de visualisation que nous possédons, le crayon sensible ne fonctionne pas en mode « écriture » [3].

2) Dans les expériences du type " coïncidences », les informations élémentaires auront été enregistrées mot par mot sur bande magnétique autonome d'un dérouleur à 16 pistes. Jusqu'à présent, ces informations, après sélection par un conditionneur câblé, étaient intégrées puis traitées par le calculateur [4]. Cependant, il s'est révélé, au cours du dépouillement de différentes expériences multiparamétriques, que ces opérations de conditionnement devaient être répétées systématiquement un grand nombre de fois; c'est pourquoi, dans l'avenir, il paraît souhaitable que ces opérations soient programmées et prises en compte par le calculateur. Dans le cadre du système CAE 510 et pour des raisons inhérentes à l'ensemble de notre installation, les informations élémentaires stockées sur bande magnétique autonome à 16 pistes seront trans- férées individuellement sur bande calculateur; on pourra, lors de ce transfert, procéder à un premier tri de ces informations et les présenter au calculateur suivant un schéma prédéterminé, afin de ne pas avoir à remanier le programme de conditionnement à l'occasion de chaque nouvelle expérience.

Une méthode fréquemment utilisée dans le cas de coïncidences entre particules chargées et rayonnement $\gamma$ consiste à tracer, après lissage, les courbes de niveau [5] de matrices $N\left(E_{\mathrm{p} . c}, E_{\gamma}\right)$. Les cartes ainsi obtenues constituent un document dont l'examen visuel permet d'obtenir des résultats qui seraient pratiquement inaccessibles par programmation. La longueur des opérations de traçage a conduit à stocker les matrices $N\left(E_{\mathrm{p} . \mathrm{c}}, E_{\gamma}\right)$ sur une bande magnétique du calculateur et à effectuer automatiquement le tracé de ces courbes de niveau, avec raccordement des cartes contiguës (ce tracé s'effectue de nuit et pendant les jours non ouvrables).

Si l'on peut considérer que l'exploitation du système est entrée dans une phase de routine, il faut cependant préciser qu'un certain nombre d'améliorations peuvent lui être apportées. Les plus importantes portent sur tout ce qui touche à l'automatisation. En particulier, dans les expériences courtes (15 à $30 \mathrm{mn}$ ), il serait souhaitable que le contrôle de l'appareillage expérimental (stabilité des pics), la conduite de l'accélérateur (changement d'énergie du faisceau), l'arrêt et le démarrage d'une nouvelle expérience soient assurés par le calculateur. Le physicien pourrait alors se consacrer au dépouillement par visualisation de l'expérience $n$ tandis que se déroulerait l'expérience $n+\mathbf{1}$. Ces modifications ne sont pas envisagées sur le système actuel, étant donné la proximité de l'arrivée d'un nouveau calculateur CII 10020.

\section{BIBLIOGRAPHIE}

[1] Bianchi (G.) et CoRge (C.), Note C.E.A., 1966, $\mathrm{n}^{\circ} \mathrm{N}-621$, p. 60.

[2] Pages (A.) et al., J. Physique, 1963, 24, 956.

[3] Daronian (D.) et Veyrié-Faucon (A.), Coll. Intern. Électron. Nucl., Versailles, 1968.
[4] BALinin (R.) et al., ce colloque, Coll. Intern. Électron. Nucl., Versailles, 1968.

[5] Kamoun (R.), Faucon (A.) et Gluzman (P.), Nucl. Instr. Methods, 1968, 58, 45-56. 